\title{
A case report of long treatment with Itraconazole in a patient with chronic Chagas disease
}

\author{
Pau Bosch-Nicolau ${ }^{1,2^{*}}$, Fernando Salvador ${ }^{1}$, Adrián Sánchez-Montalvá ${ }^{1}$, Elena Sulleiro ${ }^{3}$, Joaquín Burgos ${ }^{1}$ and \\ Israel Molina ${ }^{1 *}$ iD
}

\begin{abstract}
Background: Current available treatments (benznidazole and nifurtimox) for Chagas disease (CD) show limited efficacy in chronic phase and frequent undesirable effects. Ergosterol synthesis inhibitors (ESI) had been considered as promising drugs for CD treatment and despite its recent poor results in several clinical trials, different strategies have been proposed to optimize its role in this infection.

Case presentation: We present a case of chronic Chagas disease in patient diagnosed with HIV who received treatment for histoplasmosis with itraconazol during twelve months. Even though T. cruzi rt-PCR was persistently negative during treatment, when itraconazol was stopped she presented with a positive blood rt-PCR.

Conclusion: Several studies using different ESI had been published for CD treatment. Either in vitro or in vivo assays demonstrated activity against T. cruzi of the different triazole derivatives so different clinical trials had been carried out to evaluate its efficacy and safety. Despite contradictory evidence in the animal model, longer treatments along with other treatment strategies previously proposed suggests that ESI failure rates in positive peripheral blood rt-PCR are higher than that obtained with the current treatments of choice.
\end{abstract}

Keywords: Chagas disease, Ergosterol synthesis inhibitors, Trypanosoma cruzi

\section{Background}

Despite of the growing consensus of treating Chagas disease $(\mathrm{CD})$ in chronic stages to avoid the progression of chagasic cardiomyopathy, current available treatments (benznidazole and nifurtimox) show limited efficacy reaching cure rates between 15 and 35\% [1] and frequent undesirable effects, which leads to definitive withdrawal of the treatment in $13-32 \%$ of patients [2]. Triazole derivatives (ketoconazole, itraconazole, posaconazole, voriconazole, ravuconazole) have been used for years as antifungal treatments with a good pharmacokinetic and safety profile. They act as selective inhibitors of T. cruzi ergosterol synthesis showing potent intrinsic activity against the parasite in both in-vitro and in-vivo models, and had been considered as promising drugs for $\mathrm{CD}$ treatment [3].

\footnotetext{
*Correspondence: pau.boschnicolau@gmail.com; israelmolina@ymail.com ${ }^{1}$ Department of Infectious Diseases, Vall d'Hebrón, University Hospital, Po Vall d'Hebrón 119-129, 08035 Barcelona, Spain

Full list of author information is available at the end of the article
}

Different strategies have been proposed to optimize its outcomes in CD. Here we present a treatment failure, after the longest treatment with ergosterol synthesis inhibitors (ESI) reported in a patient with chronic CD.

\section{Case presentation}

A 38-Year-Old woman presented with a 2 months history of abdominal pain, weight loss and constipation. She was born in Bolivia (Sucre), and moved to Barcelona 7 years ago. She had no relevant pathological history. A Computed tomography $(\mathrm{CT})$ showed an ileal stenosis causing intestinal occlusion. Considering a differential diagnosis including malignancies, inflammatory disease or infectious diseases an ileum resection with termino-terminal anastomosis was carried out. The biopsy revealed the presence of a granulomatous ileitis with a PAS and Gomori stain showing fungal microorganisms and a real-time quantitative polymerase-chain-reaction (qPCR) with a positive result for Histoplasma spp. An HIV serology was done

(c) The Author(s). 2019 Open Access This article is distributed under the terms of the Creative Commons Attribution 4.0 International License (http://creativecommons.org/licenses/by/4.0/), which permits unrestricted use, distribution, and reproduction in any medium, provided you give appropriate credit to the original author(s) and the source, provide a link to the Creative Commons license, and indicate if changes were made. The Creative Commons Public Domain Dedication waiver (http://creativecommons.org/publicdomain/zero/1.0/) applies to the data made available in this article, unless otherwise stated. 
showing an advanced HIV infection with a CD4 count of $63 \mathrm{cell} / \mathrm{L}$ (7\%) and a viral load of 120,000 copies/mL. Due to the fact that was the first contact to health system a screening for imported diseases was offered including hepatitis B and C, Treponema pallidum, Strongyloides stercoralis and Trypanosoma cruzi serology and investigation for parasites in feces. Two positive serologic tests were obtained for $T$. cruzi: one of them using recombinant antigen (Bioelisa Chagas, Biokit, Lliçà d' Amunt, Spain) and the other one using a crude antigen ( $T$. cruzi ELISA, Ortho-Clinical Diagnostics, Johnson \& Johnson, High Wycombe, United Kingdom). Cardiac and gastrointestinal involvement was assessed by a 12-lead electrocardiography, chest radiography, an echocardiography, a barium enema examination, and an esophagogram showing no visceral involvement. We started antiretroviral therapy with Tenofovir/Emtricitabine and Raltegravir with rapid improve of CD4 count to normal values within 6 months. Histoplasmosis was treated with liposomal amphotericin $3 \mathrm{mg} / \mathrm{kg}$ during 10 days followed by itraconazole $200 \mathrm{mg} /$ $12 \mathrm{~h}$ during 12 months with monthly visits at the outpatient clinic assessing adherence to treatment and adverse effects. After 6 months of ESI treatment a routine qPCR [4] for T. cruzi in peripheral blood was repeated with a negative result, but after 1 year of follow-up and once treatment was stopped, a positive qPCR for $T$. cruzi was obtain. Thus, she received first line treatment for $C D$ with benznidazole $5 \mathrm{mg} / \mathrm{Kg} /$ day for 60 days with good tolerability, with yearly $T$. cruzi qPCR in peripheral blood negative during 3 years of follow-up (Fig. 1).

\section{Discussion and conclusions}

Several studies have been published using different ESI for CD treatment, initially against benznidazole resistant $T$. cruzi strains. Ketoconazole demonstrated in vitro activity against $T$. cruzi but failed to induce cure in patients with chronic phase of the disease [5]. Other ergosterol inhibitors were then tested and Venegas et al. demonstrated the effectivity of itraconazole on 20 patients with the negativization of the peripheral parasitemia in $50 \%$ of the patients using xenodiagnoses on previously positive patients and the decrease of sera lytic activity on the responder patients [6]. Urbina et al. found better cure rates with posaconazole in acute and chronic murine models compared to ketoconazole [4]. In the same line, Molina et al. tested posaconazole in a murine model of acute and chronic infection with cure rates of 90 and $60 \%$ respectively, superior than those treated with benznidazole even in immunosuppressed mice [7]. In humans, case reports of successful treatment with the triazole derivative posaconazole, have been published, even after benznidazole treatment failure in immunosuppressed patients [8]. Hence, different clinical trials had been carried out to evaluate the efficacy and safety of different triazole derivatives. In the CHAGASAZOL trial, posaconazole at different doses was compared to benznidazole in peripheral blood positive qPCR patients. Even when patients received the maximum dose approved for human use, in the intention-to-treat analysis, posaconazole showed higher treatment failure rates measured by peripheral blood qPCR of as much as $80.7 \%$ compared to a $38.4 \%$ obtained with benznidazole [9]. To come up with an optimization of treatments, after some promising combination studies performed in mice [10], the STOP-CHAGAS trial compared benznidazole and posaconazole in monotherapy and in combination. Benznidazole in monotherapy showed a parasitological cure of $86.7 \%$, higher than posaconazole in monotherapy $(13.3 \%)$ and even higher than both treatments in combination (80\%) [3]. Later on, a ravuconazole prodrug, which had a better biodisponibility and half-live, was also tested in a clinical trial. In that study, treatment with different doses of ravuconazole was compared to standard treatment with benznidazole. After a 12-month follow-up, only $29 \%$ of patients treated with high-dose ravuconazol had

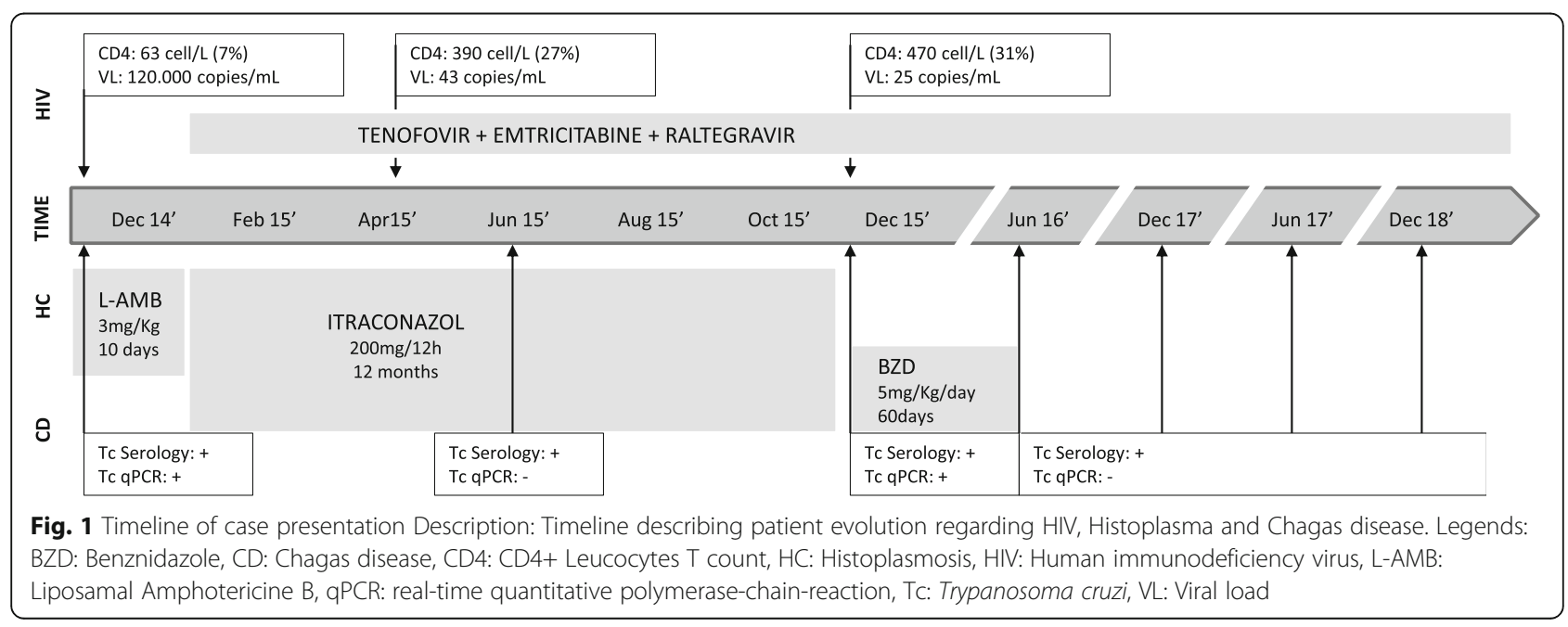


sustained parasite response compared to $82 \%$ in patients treated with benznidazole [11]. Recently, different studies testing ESI in mice using novel test of cure as the bioluminescence, showed poor results. Francisco et al. performed a chronic phase murine model whereas benznidazole showed a $100 \%$ of parasitological cure, posaconazole failed in almost all cases. These inferior cure rates were also observed when tested in the acute model when new cure standard were applied [12]. Different mechanisms were proposed to explain these results. In the late chronic stage of $C D, T$. cruzi may have quiescent amastigote forms with low replicative rates, therefore treatment using ESI, which also acts during the replicative phase, could not be enough to eliminate the parasite [13]. Additionally, it has been stated that the dose of posaconazole used in both clinical trials, although being the maximum approved in humans, would suppose a drug exposure of 10 to $20 \%$ of the cure dose administered in mice [14]. Hence, longer treatments with ESI have been suggested to overcome that lack of efficacy. The patient presented here received twelve months of itraconazole to treat an ileal histoplasma infection. However, erratic drug absorption and distribution could have occurred since drug levels during treatment were not obtained. Although little evidence is derived from a single case, our patient showed a positive qPCR persistence in peripheral blood at the end of treatment, which is in line with a previous case published by Galhardo et al. [15] and previous murine models suggesting scarce benefit of prolonged ESI treatment [16]. In our opinion, despite the contradictory evidence of ESI in the animal model, all published data including our case suggests that ESI failure rates in positive peripheral blood qPCR are higher than that obtained with the current treatments of choice. Due to the fact that HIV patients are at risk of reactivation, prompt etiological treatment and close follow-up should be guaranteed.

\section{Abbreviations}

CD: Chagas disease; ESI: Ergosterol synthesis inhibitors; qPCR: real-time quantitative polymerase-chain-reaction

\section{Acknowledgements}

Not applicable.

\section{Authors' contributions}

PB-N, FS, AS-M, JB and IM analyzed and interpreted the patient data regarding Chagas disease and its treatment. ES performed the microbiological diagnosis. PB-N wrote the manuscript. All authors read and approved the final manuscript.

\section{Funding}

This research received no specific grant from any funding agency in the public, commercial, or not-for-profit sector.

\section{Availability of data and materials}

Data sharing is not applicable to this article as no datasets were generated or analysed during the current study.

\section{Ethics approval and consent to participate}

The study was approved by the Ethics Committee of Vall d'Hebron Research Institute.

\section{Consent for publication}

The patient gave written consent for their personal or clinical details along with any identifying images to be published in this study.

\section{Competing interests}

The authors declare that they have no competing interests.

\section{Author details}

'Department of Infectious Diseases, Vall d'Hebrón, University Hospital, po Vall d'Hebrón 119-129, 08035 Barcelona, Spain. ²Department of Medicine, Universitat Autònoma de Barcelona, Barcelona, Spain. ${ }^{3}$ Department of Microbiology, Vall d'Hebron, University Hospital, PROSICS Barcelona, Barcelona, Spain.

Received: 6 June 2019 Accepted: 30 October 2019

Published online: 09 November 2019

\section{References}

1. Viotti R, Vigliano C, Lococo B, et al. Long-term cardiac outcomes of treating chronic Chagas disease with benznidazole versus no treatment: a nonrandomized trial. Ann Intern Med. 2006;144(10):724-34.

2. Molina I, Salvador F, Sánchez-Montalvá A, Treviño B, Serre N, Sao Avilés A, Almirante B. Toxic profile of Benznidazole in patients with chronic Chagas disease: risk factors and comparison of the product from two different manufacturers. Antimicrob Agents Chemother. 2015;59(10):6125-31.

3. Urbina JA, Payares $\mathrm{G}$, Contreras $\mathrm{LM}$, et al. Antiproliferative effects and mechanism of action of SCH 56592 against Trypanosoma (Schizotrypanum) cruzi: in vitro and in vivo studies. Antimicrob Agents Chemother. 1998;42(7):1771-7.

4. Piron M, Fisa R, Casamitjana N, et al. Development of a real-time PCR assay for Trypanosoma cruzi detection in blood samples. Acta Trop. 2007;103:195-200.

5. Brener Z, Cançado JR, Galvão LM, et al. An experimental and clinical assay with ketoconazole in the treatment of Chagas disease. Mem Inst Oswaldo Cruz. 1993;88(1):149-53.

6. Venegas J, Zulantay I, Apt W, Solari A, Díaz E, Sánchez G. Effect of itraconazole on lytic antibodies levels in chronic Chagas disease. Parasite. 1997;4(3):259-62.

7. Molina J, Martins-Filho O, Brener Z, Romanha AJ, Loebenberg D, Urbina JA. Activities of the triazole derivative SCH 56592 (posaconazole) against drugresistant strains of the protozoan parasite Trypanosoma (Schizotrypanum) cruzi in immunocompetent and immunosuppressed murine hosts. Antimicrob Agents Chemother. 2000;44(1):150-5.

8. Pinazo MJ, Espinosa G, Gallego M, Lopez-Chejade PL, Urbina JA, Gascon J. Successful treatment with Posaconazole of a patient with chronic Chagas disease and systemic lupus Erythematosus. Am J Trop Med Hyg. 2010;82(4):583-7.

9. Molina I, Gómez i Prat J, Salvador F, et al. Randomized trial of Posaconazole and Benznidazole for chronic Chagas' disease. N Engl J Med. 2014;370(20):1899-908.

10. de DinizL F, Urbina JA, de Andrade IM, et al. Benznidazole and posaconazole in experimental Chagas disease: positive interaction in concomitant and sequential treatments. PLoS Negl Trop Dis. 2013;7(8):e2367.

11. Torrico F, Gascon J, Ortiz L, et al. Treatment of adult chronic indeterminate Chagas disease with benznidazole and three E1224 dosing regimens: a proof-ofconcept, randomised, placebo-controlled trial. Lancet Infect Dis. 2018;18:419-30.

12. Francisco AF, Lewis MD, Jayawardhana S, Taylor MC, Chatelain E, Kelly JM. Limited ability of posaconazole to cure both acute and chronic Trypanosoma cruzi infections revealed by highly sensitive in vivo imaging Antimicrob Agents Chemother. 2015;59(8):4653-61.

13. Sánchez-Valdéz FJ, Padilla A, Wang W, Orr D, Tarleton RL. Spontaneous dormancy protects Trypanosoma cruzi during extended drug exposure. Elife. 2018;26:7.

14. Urbina JA. Recent clinical trials for the etiological treatment of chronic chagas disease: advances, challenges and perspectives. J Eukaryot Microbiol. 2015:62(1):149-56

15. Galhardo MC, Martins IA, Hasslocher-Moreno A, et al. Reactivation of Trypanosoma cruzi infection in patients with acquired immunodeficiency syndrome. Rev Soc Bras Med Trop. 1999;32(3):291-4.

16. Khare S, Liu X, Stinson M, et al. Antitrypanosomal treatment with benznidazole is superior to posaconazole regimens in mouse models of Chagas disease. Antimicrob Agents Chemother. 2015;59(10):6385-94.

\section{Publisher's Note}

Springer Nature remains neutral with regard to jurisdictional claims in published maps and institutional affiliations. 